\title{
Aplicación del índice de riesgo nutricional geriátrico en la unidad de cuidados intensivos de la clínica La Merced, Quito Ecuador.
}

Application of the geriatric nutritional risk index in the intensive care unit of the La Merced clinic, Quito, Ecuador.

Vinueza Ortiz Diana. ${ }^{1}$, Noboa Escobar Diego Mauricio. ${ }^{2} \&$ Salome Shayana Ruales Paredes. $^{3}$

\begin{abstract}
.
DOI: $\underline{\text { https://doi.org/10.33262/cienciadigital.v3i1.279 }}$

The aim of implementing the Geriatric Nutritional Risk Index (GNRI) was to predict the likelihood of developing malnutrition adverse consequences, such as a increased risk of infection, hospital stay and mortality in critical elderly patients and to observe its behavior in the pathologies of chronic liver damage and severe kidney damage.

This paper presents analyses of cross-sectional data collected from 61 patients older than 60 years admitted to the Critical Care Unit during the May-August 2013 period. Using inferential statistics, it was possible to make a multiple factor analysis to determine the association between GNRI, survival rate, development of infectious processes and the time of hospitalization The GNRI achieved to predict hospital mortality, however, infection rates and hospitalization period were related to factors such as admitting diagnosis and high exposure to infectious agents. To sum up, it was not possible to associate GNRI and the pathologies aforementioned, nevertheless, in order to obtain conclusive results, is necessary to apply the GNRI to a wider sample of patients.
\end{abstract}

Keywords: Nutrition Index, mortality, morbidity, survival rate.

1 Universidad Católica del Ecuador, Quito, Ecuador, dianavizuela@gmail.com

2 Hospital Pablo Arturo Suarez, Gestión de Docencia e Investigación, Quito, Ecuador, diego.noboa@hpas.gob.ec.

3 Universidad Central de Ecuador, Facultad de Ciencias de la discapacidad, Quito, Ecuador, salomerualesparedes@gmail.com 


\section{Resumen.}

La aplicación del Índice de Riesgo Nutricional Geriátrico (IRNG) tuvo como finalidad la predicción de la probabilidad a desarrollar consecuencias adversas a la desnutrición como aumento del riesgo de infección, estancia hospitalaria y mortalidad en los pacientes críticos de tercera edad; y observar su comportamiento en las patologías de daño hepático crónico y daño renal severo. La presente investigación tiene un carácter observacional y analítico longitudinal. Se incluyeron a 61 pacientes mayores de 60 años de la Unidad de Cuidados Intensivos (UCI) de la Clínica "La Merced" (Quito, Ecuador), durante los meses de mayo a agosto del 2013. A través de la estadística inferencial se realizó un análisis multifactorial de correspondencias múltiples y desagregaciones para determinar la asociación entre el IRNG, la sobrevida, el desarrollo de un proceso infeccioso y el tiempo de estancia hospitalaria.

El IRNG pudo pronosticar la mortalidad, sin embargo, la presencia de infección y estancia hospitalaria dependieron de factores como el diagnóstico de ingreso, y la alta exposición a agentes infecciosos.

No se observó una asociación concluyente entre las patologías anteriormente mencionadas y el IRNG, no obstante, se requiere de la realización de estudios con una muestra mayor para confirmar la aplicación del IRNG en todos los pacientes geriátricos.

Palabras claves: Índice Nutricional, morbilidad, mortalidad, supervivencia.

\section{Introducción.}

Aproximadamente el 50\% de los pacientes atendidos en la Unidad de Cuidados Intensivos de la Clínica "La Merced" corresponden a la tercera edad. Debido a la avanzada edad, este grupo etario se encuentran expuesto a condiciones fisiológicas que aumentan su probabilidad de desnutrición, manifestándose la necesidad de la aplicación de un índice que pueda predecir la probabilidad de morbilidad y mortalidad.

Montejano, L (2012) señala que el índice de riesgo nutricional geriátrico fue creado en 1991, como una variación del índice de riesgo nutricional debido a que en el adulto mayor la obtención del peso usual no era posible en la mayoría de los casos. [1] El IRNG utiliza la albúmina y el peso ideal de Lorentz, se basa en la altura y sexo del paciente dando como resultado la siguiente ecuación $($ IRNG $=[1.489 \mathrm{x}$ albumina $(\mathrm{g} / \mathrm{L})]+[41.7 \mathrm{x}($ peso/PLo) $]$ ). Esta fórmula permite clasificar al paciente en 4 grados que determinan el riesgo de morbilidad y mortalidad; riesgo alto: (IRNG < 82), riesgo moderado: (IRNG 82-91), riesgo bajo: (IRNG 92 y menor o igual 98), sin riesgo: (IRNG >98).

En la Unidad de Cuidados Intensivos las herramientas para determinar la situación nutricional del paciente como la Valoración Global Subjetiva y el Mini Nutritional 
Assesment (MNA) no son aplicables debido al Glasgow con el que se encuentran la gran mayoría de individuos, de esta forma el IRNG constituye un instrumento de gran utilidad para la identificación de pacientes, que se encuentran en riesgo de desnutrición y que requieren soporte nutricional debido al estrés metabólico.

\section{Objetivo:}

El principal propósito de esta investigación es evaluar la efectividad del índice de riesgo nutricional geriátrico para la predicción de la probabilidad de mortalidad y morbilidad del adulto mayor en la Unidad de Cuidados Intensivos de la Clínica "La Merced” y su asociación con variables tales como la estancia hospitalaria, desarrollo de procesos infecciosos, sobrevida y patologías como daño renal crónico y daño hepático severo.

\section{Metodología:}

El presente estudio posee un enfoque cuantitativo, observacional analítico longitudinal. Se observó el comportamiento del IRNG como predictor de mortalidad y morbilidad del paciente geriátrico, desde el ingreso del paciente a UCI hasta su salida. La Clínica "La Merced" atiende cerca de 400 pacientes al año de los cuales aproximadamente el 50\% pertenecen a la tercera edad. La muestra fue obtenida mediante la recolección de datos de las historias clínicas de los pacientes de la tercera edad durante los meses de Mayo a Agosto del 2013. Fueron incluidos aquellos pacientes cuya edad fue mayor a 60 años y su estadía fue mayor a 24 horas, fueron excluidos aquellos sujetos que no cumplieron con lo antes citado.

Las variables se analizaron a través de estadística descriptiva utilizando la distribución porcentual para expresar las características demográficas, estado nutricional y comportamiento de albúmina e índice de riesgo nutricional, presencia de procesos infecciosos, estancia hospitalaria y diagnóstico de ingreso.

Se utilizó la estadística inferencial mediante un análisis factorial de correspondencias múltiples y desagregaciones con el propósito de poner en evidencia la asociación entre el IRNG y variables tales como, daño hepático crónico, daño renal severo, presencia de procesos infecciosos, estadía, sobrevida, estado nutricional. Mediante la aplicación del test de Fisher se analizaron las variables de sobrevivencia a la Unidad de Cuidados Intensivos y Estado Nutricional.

\section{Resultados:}

El 32.8\% de la población no presentó ningún grado de IRNG el 16.4\% presentó un bajo IRNG, 32.8\% moderado IRNG, mientras que el 18\% desarrolló un alto IRNG. El $41 \%$ de la población total sobrevivió a UCI. 
Se observó una asociación entre los pacientes sin riesgo nutricional geriátrico y la sobrevivencia a UCI, de igual forma se deduce una asociación evidente con la no sobrevivencia a los pacientes que tienen IRNG moderado. Se debe considerar que el porcentaje de pacientes que se encontraron en moderado riesgo fue mayor al de los pacientes con alto riesgo. (Figura $\mathrm{N}^{\circ} 1$ ).

Figura 1. Mapa perceptual de asociaciones entre las variables: clasificación de IRNG, número de días de estadía y sobrevivencia a la Unidad de Cuidados Intensivos de los pacientes evaluados en la Clínica "La Merced", Quito, Ecuador, durante el periodo de Mayo-Agosto 2013.

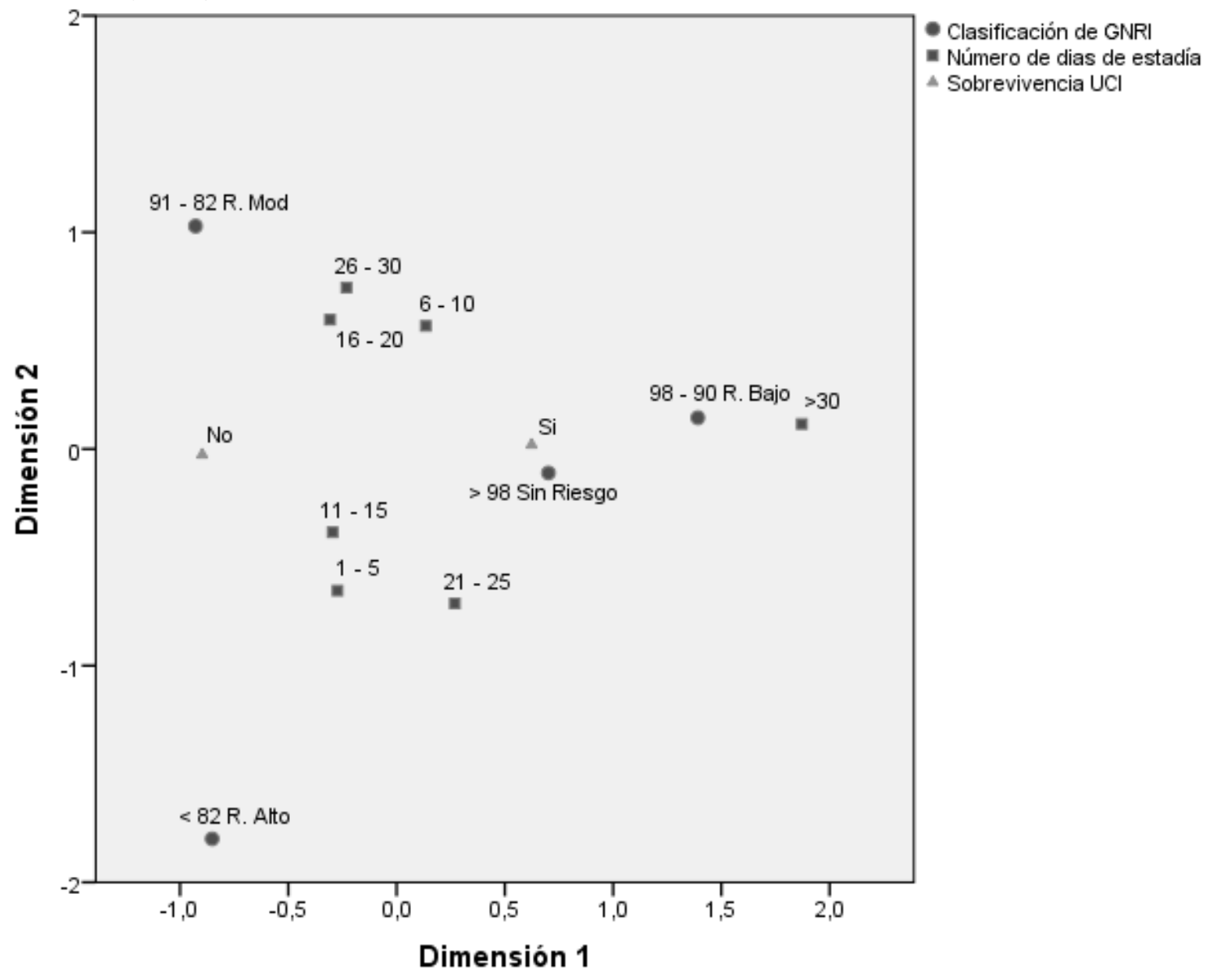

Fuente: Clínica "La Merced” durante el periodo Mayo-Agosto 2013 Elaborado por: Diana Vinueza

La estadía del paciente no se asocia de forma objetiva con el índice de riesgo nutricional. La relación a mayor IRNG menos estadía, se puede explicar porque estos pacientes fallecieron debido a la complejidad del cuadro clínico de ingreso. (Figura 1).

Se observa una asociación entre el Diagnóstico Nutricional y la Sobrevivencia UIC ( $\mathrm{p}<$ 0,01 ); siendo el $41 \%$ de personas que fallecieron, de los cuales el $24,6 \%$ presentaban algún 
grado de desnutrición desnutrición. Esta situación es ocasionada debido a las modificaciones morfológicas y fisiológicas que ocurren en un individuo conforme va avanzando la edad como son disfagia, edentulismo, atrofia de las vellosidades, modificaciones en el gusto y otras, el anciano tiene un alto riesgo de desnutrición y se sufrir las consecuencias adversas a la misma. Es importante también mencionar los determinantes sociales del paciente geriátrico como son la dependencia de terceros, el abandono y otras que juegan un papel importante en el estado nutricional. (Tabla 1).

Tabla 1. Desagregación del estado nutricional del adulto mayor con respecto a la sobrevivencia a la Unidad de Cuidados Intensivos en los pacientes evaluados en la Clínica “La Merced”, Quito, Ecuador, durante el periodo de Mayo-Agosto 2013.

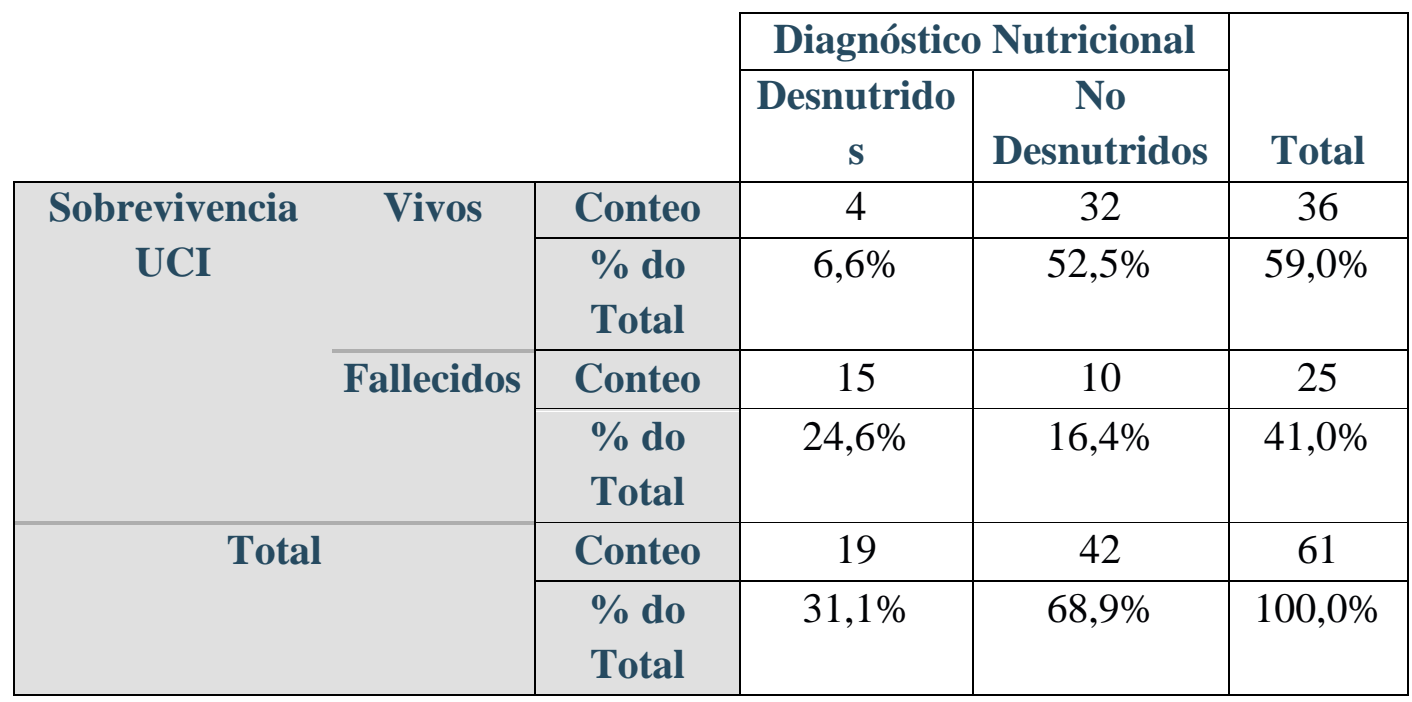

Elaborado por: Diana Vinueza

La asociación entre el índice de riesgo nutricional geriátrico y la presencia de daño renal crónico no es objetiva, por lo tanto, se deduce que son variables independientes. De igual forma se observó que el daño hepático no caracteriza al IRNG. (Figura $N^{\circ} 2$ ).

Figura 2. Mapa perceptual de asociaciones entre las variables: clasificación de IRNG, pacientes con daño hepático crónico, pacientes con daño renal crónico, presencia de infección y sobrevivencia correspondiente a los pacientes evaluados en la Clínica “La Merced” durante el periodo de Mayo-Agosto 2013. 


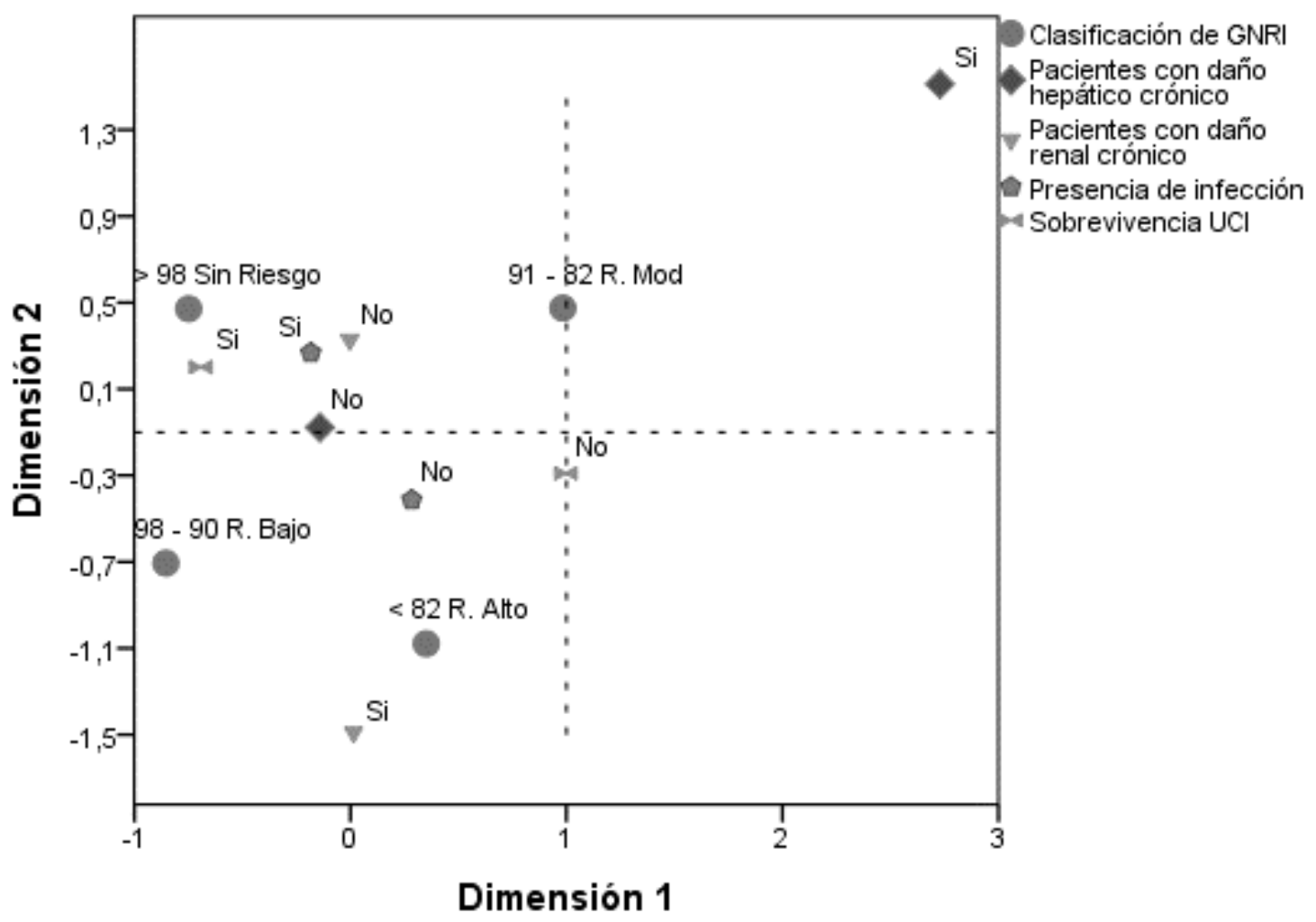

Fuente: Clínica "La Merced” durante el periodo Mayo-Agosto 2013

Elaborado por: Diana Vinueza

\section{Discusión:}

El adulto mayor está predispuesto a la desnutrición y consecuentemente a aumentar su estancia hospitalaria, la susceptibilidad a infecciones, dehiscencia de heridas e incrementar la probabilidad de mortalidad.

Poma \& Cols. (2012) en su estudio realizado en Lima, donde fueron incluidos 279 individuos, mencionan que el promedio de estadía en el paciente geriátrico crítico oscila entre $7.4 \pm 6.8$ días, siendo este un periodo de tiempo donde el catabolismo proteico deteriora el estado nutricional del individuo; la mortalidad correspondió al 29\%. [2]

Charlton \& Cols. (2012) realizaron un estudio en Australia, donde fueron incluidos 774 pacientes mayores a 65 años para determinar la prevalencia de la desnutrición, utilizando el Mini Nutritional Assesment, éste fue del 55\% y la mortalidad correspondió al 14\%, posteriormente se determinó que la desnutrición y el riesgo de desnutrición en el adulto mayor es un factor que aumenta su mortalidad. [3]

A lo largo de los últimos años se han desarrollado varios estudios acerca de la efectividad del IRNG como herramienta para predecir la mortalidad. Cereda \& Cols. (2006) realizaron un estudio en Italia, con la finalidad de validar el IRNG como indicador de mortalidad, en 
un corto periodo de tiempo en pacientes geriátricos hospitalizados. Se realizó el seguimiento durante un mes, el $4.6 \%$ de los pacientes fallecieron y consecuentemente se encontraron con un IRNG < 92. [4]. Posteriormente se lo ha comparado con otras herramientas para la predicción de desnutrición como en el estudio realizado por Cereda \& Cols. (2011) donde se aplicaron el MNA y el IRNG; fueron excluidos aquellos pacientes que cruzaban por enfermedades terminales y desórdenes neoplásicos. El 77.5\% de la población se encontró con un IRNG menor a 92 y que este grupo tenía una significativa relación $<0,05$ con la mortalidad. [5]

La desnutrición ocasiona efectos trascendentales en el sistema inmunológico; un paciente hipercatabólico que se encuentre en un ambiente de riesgo microbiológico tiene una gran posibilidad de iniciar un proceso infeccioso. Lee \& cols. (2012) realizaron un estudio observacional en Corea, éste incluyó a 401 pacientes con sepsis. El 12.7\% de sujetos falleció y se encontraron con un IRNG menor a 87. [6]

En el presente estudio se puede observar como un IRNG $<91$ se asocia con la mortalidad y la ausencia de riesgo nutricional se asocia a la sobrevida, sin embargo, el desarrollo de proceso infecciosos dependió de otros factores como la alta exposición a agentes infecciosos en UCI.

Existen varios estudios acerca de la aplicación del IRNG, principalmente en los realizados por Cereda \& cols. y Bouillanne \& cols. se menciona que es necesario utilizar como criterios de exclusión a las patologías renales crónicas, hepático severas, trastornos neoplásicos e infección severa, debido a que en estos cuadros clínicos la concentración de albúmina sérica se altera sin deberse a causas de etiología nutricional. Se observó el comportamiento del Índice de Riesgo Nutricional Geriátrico en las patologías de daño renal crónico, daño hepático severo, hipernatremia e hiponatremia y se determinó que éstas no caracterizan al IRNG. En esta investigación no se excluyó a patologías como daño hepático crónico y daño hepático severo, se determinó que éstas no caracterizan al IRNG. [7]

\section{Conclusiones}

- En el presente estudio, el IRNG pudo pronosticar la presencia de infección y estancia hospitalaria, sin embargo, éstos dependieron de factores como el diagnóstico de ingreso, y la alta exposición a agentes infecciosos en UCI.

- Debido a modificaciones morfológicas y funcionales, la tercera edad es un grupo etario con alta predisposición a la desnutrición, el alto estrés metabólico y la probabilidad de sufrir infecciones intrahospitarias pueden aumentar la mortalidad; consecuentemente se evidenció asociación entre el estado nutricional y la sobrevivencia a la Unidad de Cuidados Intensivos, hecho que muestra la importancia de la evaluación, seguimiento y soporte nutricional. 
- Se observó el comportamiento del Índice de Riesgo Nutricional Geriátrico en las patologías de daño renal crónico, daño hepático severo, hipernatremia e hiponatremia y se determinó que éstas no caracterizan al IRNG, es necesario mencionar que la cantidad de pacientes en cada patología puede no haber sido representativas y es necesario realizar el estudio con una muestra de mayor tamaño.

\section{Referencias Bibliográficas.}

[7] Bouillanne, O., Morineau, G., Dupont, C., Coulombel, I., Vincent, J.P., Nicolis, I., Benazeth, S., Cynober, L. \& Aussel, C. (2005). Geriatric Nutritional Risk Index: a new index for evaluating at-risk elderly medical patients [Versión Electrónica]. The American Journal of Clinical Nutrition. Vol. 82 ( $\left.\mathrm{N}^{\circ} 4\right), 777-783$.

[4] Cereda, E., Limonta, D., Pusani, C. \& Vanotti, A. (2006) Geriatric nutritional risk index: A posible indicator of short-term mortality in acutely hospitalized older people. [Versión Electrónica]. Letters to the editor. Vol. 54 ( $\mathrm{N}^{\circ}$ 6) 1011-1012.

[5] Cereda, E., Pedrolli C., Zagami, A., Vanotti, A., Piffer S., Opizzi A., Rodanelli M., Cacciallannza R., (2011) Nutritional screening and mortality in newly institutionalised elderly: a comparison between the geriatric nutritional risk index and the mini nutritional assessment. [Versión Electrónica]. Clinical Nutrition . Vol. $30\left(\mathrm{~N}^{\circ}\right.$ 6) 793-798.

[3] Charlton, K., Batterham, M., Bowden, S., Ghosh, A., Caldwel, K., Barone, L., Mason, M., Potter, J., Ayer, B., \& Milosavljevic, M. (2013) A high prevalence of malnutrition in acute geriatric patients predicts adverse clinical outcomes and mortality within 12 months. e-SPEN Journal. e120-e125. Consultado el 28 de diciembre, $2013 . \quad$ En: http://www.espenjournal.org/article/PIIS2212826313000237/fulltext

[6] Lee, J., Hong, C., Kwon, O., Hong, H., Ko, Y. (2012). Performance of the geriatric nutritional risk index for predicting the 28-day in-hospital mortality in older patients with sepsis presenting to the emergency department. [Versión Electrónica]. Revista Elsevier. Vol. 7. (N²), 272

[1] Montejano, R. (2012). Evaluación de riesgo nutricional y de factores asociados en adultos mayores no institucionalizados en la provincia de Valencia. Tesis Doctoral no publicada, Universidad de Alicante, Departamento de Enfermería, Alicante, España.

[2] Poma, J., Gálvez, M., Zegarra, J., Meza, M., Varela, L. \& Chávez, H. (2012). Morbimortalidad de pacientes mayores de 60 años en el servicio de cuidados intensivos de un hospital general. [Versión Electrónica]. Revista Médica Herediana. Vol. $23\left(\mathrm{~N}^{\circ} 1\right), 16-22$.

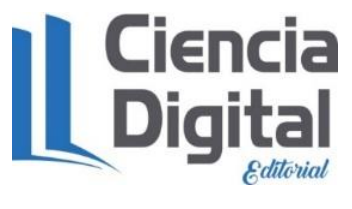




\section{Para citar el artículo indexado.}

Vinueza D, Noboa D. \& Ruales S. (2019). Aplicación del índice de riesgo nutricional geriátrico en la unidad de cuidados intensivos de la clínica La Merced, Quito Ecuador. Revista electrónica Ciencia Digital 3(1), 180-189. Recuperado desde: http://cienciadigital.org/revistacienciadigital2/index.php/CienciaDigital/article/view/279/67 $\underline{3}$

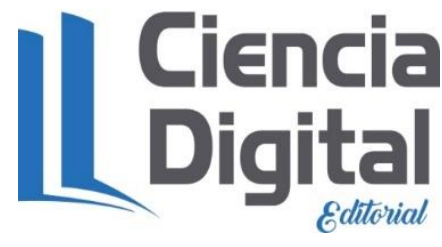

El artículo que se publica es de exclusiva responsabilidad de los autores y no necesariamente reflejan el pensamiento de la Revista Ciencia Digital.

El artículo queda en propiedad de la revista y, por tanto, su publicación parcial y/o total en otro medio tiene que ser autorizado por el director de la Revista Ciencia Digital.
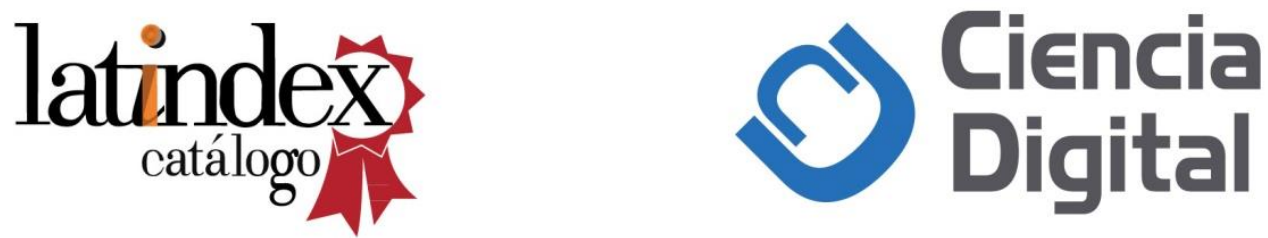